\title{
Hop2 and Sae3 Are Required for Dmc1-Mediated Double-Strand Break Repair via Homolog Bias during Meiosis
}

\author{
Hong-Rae Cho ${ }^{1,2}$, Yoon-Ju Kong ${ }^{1,2}$, Soo-Gil Hong ${ }^{1,2}$, and Keun Pil Kim, *
}

\begin{abstract}
During meiosis, exchange of DNA segments occurs between paired homologous chromosomes in order to produce recombinant chromosomes, helping to increase genetic diversity within a species. This genetic exchange process is tightly controlled by the eukaryotic RecA homologs Rad51 and Dmc1, which are involved in strand exchange of meiotic recombination, with Rad51 participating specifically in mitotic recombination. Meiotic recombination requires an interaction between homologous chromosomes to repair programmed double-strand breaks (DSBs). In this study, we investigated the budding yeast meiosisspecific proteins Hop2 and Sae3, which function in the Dmc1-dependent pathway. This pathway mediates the homology searching and strand invasion processes. Mek1 kinase participates in switching meiotic recombination from sister bias to homolog bias after DSB formation. In the absence of Hop2 and Sae3, DSBs were produced normally, but showed defects in the DSB-to-single-end invasion transition mediated by Dmc1 and auxiliary factors, and mutant strains failed to complete proper chromosome segregation. However, in the absence of Mek1 kinase activity, Rad51dependent recombination progressed via sister bias in the hop2 $\Delta$ or sae3A mutants, even in the presence of Dmc1. Thus, Hop2 and Sae3 actively modulate Dmc1-dependent recombination, effectively progressing homolog bias, a process requiring Mek1 kinase activation.
\end{abstract}

\section{INTRODUCTION}

Meiosis employs two rounds of the cell division to produce haploid gametes (sperms or eggs), which are essential for sexual reproduction. During meiosis, programmed genetic recombination generates genetic diversity in organisms and ensures faithful chromosome segregation. Recombination events are

${ }^{1}$ Department of Life Sciences, Chung-Ang University, Seoul 06974, Korea, ${ }^{2}$ These authors contributed equally to this work.

${ }^{*}$ Correspondence: kpkim@cau.ac.kr

Received 28 March, 2016; revised 10 May, 2016; accepted 12 May, 2016; published online 21 June, 2016

Keywords: budding yeast, double-strand breaks, Hop2, recombination, Sae3 initiated by meiosis-specific programmed double-strand break (DSB) formation regulated by the topoisomerase-like protein Spo11 (Keeney, 2001). DSBs undergo an additional process to expose $3^{\prime}$ single stranded DNA (ssDNA), which is mediated by the Exo1/Dna2 and Mre11/Rad50/Xrs2 complex (Cannavo and Cejka, 2014; Garcia et al., 2011). DSB ends with 500 nucleotide (nt) $3^{\prime}$ ssDNA tails preferentially interact with the homolog partner template rather than with the sister chromatid; these reactions are mediated by the RecA homolog proteins Dmc1 and Rad51 (Hong et al., 2013a; Lao et al., 2013). Dmc1 is a meiosis-specific recombinase found in most eukaryotes, including yeast, mice, and humans. Rad51 functions in homology searching and homolog pairing of DNAs during mitosis, but plays an auxiliary role in homology searching associated with Dmc1 during meiosis (Hong et al., 2013a). The dmc1s mutant in budding yeast shows an abnormal phenotype during recombination that reflects the essential role of this protein in meiotic recombination. A Dmc1-deficient strain exhibited defective DSB repair progression and deficient synaptonemal complex formation (Shinohara et al., 1997; Schwacha and Kleckner, 1997). It has been suggested that Dmc1 promotes DNA strand exchange to form synapsis exclusively between homologs, which is a process unique to meiotic recombination. In addition, DSBs accumulate in $d m c 1 \Delta$ mutants because of failed strand invasion, which triggers a checkpoint during meiotic prophase I. It has been reported that the Hop2-Mnd1 and Mei5-Sae3 complexes are dominant auxiliary factors for Dmc1 function and stimulate Dmc1-mediated ssDNA strand invasion during meiotic recombination (Chan et al., 2014; Ferrari et al., 2009). The Hop2Mnd1 heterodimeric complex constructs a synaptic complex, which is a combination of $3^{\prime}$ ssDNA and homologous doublestrand DNA in the D-loop formation stage (Chi et al., 2007; Kang et al., 2015; Pezza et al., 2007). Previous studies showed that in Saccharomyces cerevisiae, hop2 $\Delta$ or sae34 mutant exhibits prophase arrest and DSB accumulation (Henry et al., 2006; Leu et al., 1998; Tsubouchi and Roeder, 2002). The Mei5Sae3 heterodimeric complex is specifically expressed in meiotic cells (Hayase et al., 2004; Tsubouchi and Roeder, 2004). mei5s or sae34 mutant exhibits inefficient spore viability, prophase arrest, and defective recombinant products (Hayase et al., 2004; Tsubouchi and Roeder, 2004). Biochemical studies have shown that the Mei5-Sae3 complex promotes nucleation of Dmc1 onto ssDNA (Tsubouchi and Roeder, 2004). Therefore, the Hop2-Mnd1 and Mei5-Sae3 complexes may be essential for homolog bias mediated by Dmc1-dependent recombination

eISSN: 0219-1032

(c) The Korean Society for Molecular and Cellular Biology. All rights reserved.

(c) This is an open-access article distributed under the terms of the Creative Commons Attribution-NonCommercial-ShareAlike 3.0 Unported License. To view a copy of this license, visit http://creativecommons.org/licenses/by-nc-sa/3.0/. 
in early prophase I.

Mek1 kinase is expressed specifically during meiosis and is activated by the induction of DSBs (Niu et al., 2007). Mek1 kinase activity inhibits inter-sister DSB repair during meiosis by directly affecting the sister chromatid axes. The pattern of meiotic recombination by Dmc1 and Rad51 shows that Mek1 kinase involves in Rad51-mediated recombination (Hong et al., 2013; Lao et al., 2013). Related studies revealed that Rad51' strand exchange activity is inhibited by tightly controlled mechanisms that prevent the complex formation between Rad51 and Rad54 during meiotic recombination (Busygina et al., 2008). Thus, meiosis-specific Hed1 binds to Rad51, blocking the interaction with Rad54 and decreasing the binding affinity of Rad54 for Rad51 by phosphorylating Rad54 through Mek1 (Busygina et al., 2008; Niu et al., 2009). The choice of a partner for recombination during meiosis is highly modulated by interhomolog repair pathways after DSB resection to expose the $3^{\prime}$ end. Partner choice is modulated by Red1, Hop2, and Mek1 kinase activity (Hong et al., 2013a; Kim et al., 2010; Schwacha and Kleckner, 1997). Inactivation of Mek1 kinase promotes DSB repair to progress efficiently even in the absence of Dmc1; however, loss of homolog bias results in the repair of most DSBs via sister bias. In the absence of Mek1 kinase activity, cohesin channels the recombination to use the sister chromatid as a template, which is appropriate for mitotic DSB repair (mitotic mode) (Hong et al., 2013a). Thus, the sister chromatid is preferentially used as a partner template. This meiosis-specific pathway promotes homolog bias during the early stages post DSB, and additional pathways support that the bias is maintained during the interhomolog single-end invasion (IH-SEI) to interhomolog double-Holliday junctions $(\mathrm{IH}-\mathrm{dHJ})$ transition of interhomolog crossover (IH-CO) formation (Kim et al., 2010). The Hop2-Mnd1 complexes stimulate Dmc1-mediated strand exchange (Petukhova et al., 2005). Additionally, the Mei5-Sae3 complex co-localizes with Dmc1 and is required for Dmc1 function in vivo (Hayase et al., 2004; Tsubouchi and Roeder, 2004).

In this study, we investigated the roles of Hop2 and Sae3 during recombination in the presence or absence of Mek1 kinase activity, and found that both Hop2 and Sae3 promote homolog bias through a Dmc1-dependent pathway.

\section{MATERIALS AND METHODS}

\section{Yeast strains}

Saccharomyces cerevisiae strains used in this study included SK1 derivatives isogenic with homozygous ho::hisG, leu2::hisG, ura3A (Pst1-Sma1). All genotypes and strain details are described in Supplementary Table 1.

\section{Culture media and time course}

The culture media and meiotic time course were essentially performed as previously described (Kim et al., 2010). Cells were patched to YPG plates (1\% yeast extract, $2 \%$ peptone, $3 \%$ glycerol, and $2 \%$ bactoagar) for $24 \mathrm{~h}$ at $30^{\circ} \mathrm{C}$. To select single colonies, cells from the YPG plate were streaked onto YPD plates (1\% yeast extract, $2 \%$ peptone, $2 \%$ glucose, and $2 \%$ bactoagar) and grown at $30^{\circ} \mathrm{C}$ for 2 days. A single diploid colony resulting from this streaking was inoculated into $2 \mathrm{ml}$ liquid YPD medium (1\% yeast extract, $2 \%$ peptone, $2 \%$ glucose) and incubated at $30^{\circ} \mathrm{C}$ for $24 \mathrm{~h}$. For synchronous meiosis, YPD cultures were inoculated in SPS medium (1\% potassium acetate, $1 \%$ bactopeptone, $0.5 \%$ yeast extract, $0.17 \%$ yeast nitrogen without amino acids, $0.5 \%$ ammonium sulfate, $0.05 \mathrm{M}$ potassium biphthalate, and 2 drops/L antifoam [Sigma, USA],
$\mathrm{pH} 5.5)$ at a 1:500 dilution and cultured for $18 \mathrm{~h}$. Meiosis was initiated in SPM medium $(0.2 \%$ potassium acetate, $0.02 \%$ raffinose, and 2 drops/L antifoam). Meiotic cells were harvested and resuspended in $50 \mathrm{mM}$ Tris- $\mathrm{HCl}$ and $50 \mathrm{mM}$ EDTA. Crosslinking of cells was performed with psoralen under UV light for $10 \mathrm{~min}$.

\section{Physical analysis of meiotic recombination}

Cultured cells at each time point were treated with Zymolyase (100T, US Biological, USA). Spheroplasted cells were subjected to guanidine-phenol extraction. DNA was precipitated with ethanol, and then resuspended in $50 \mathrm{mM}$ Tris- $\mathrm{HCl}$ and $1 \mathrm{mM}$ EDTA (Hong et al., 2013b; Kim et al., 2010). DNA concentration was measured using a Picogreen assay kit (Invitrogen, USA). For one-dimensional gel analysis, $2 \mu \mathrm{g}$ genomic DNA was treated with Xhol (Enzynomics, Korea) for $3 \mathrm{~h}$. Next, the DNA samples were loaded into a $0.6 \%$ Seakem LE agarose gel in TBE buffer at $\sim 2 \mathrm{~V} / \mathrm{cM}$ for $24 \mathrm{~h}$. For two-dimensional gel analysis, DNA digested in the same manner was then loaded into a $0.4 \%$ Seakem Gold agarose gel without ethidium bromide in TBE buffer at $\sim 1 \mathrm{~V} / \mathrm{cM}$ for $21 \mathrm{~h}$. After electrophoresis, the gel was stained for $30 \mathrm{~min}$ with $0.5 \mu \mathrm{g} / \mathrm{ml}$ ethidium bromide. Bands of interest were cut and arrayed on $2 \mathrm{D}$ gel trays. The gel was loaded in a two-dimensional manner. SeaKem LE agarose $(0.8 \%)$ containing $0.5 \mu \mathrm{g} / \mathrm{ml}$ ethidium bromide was poured around the cut $1 \mathrm{D}$ gel array at $4^{\circ} \mathrm{C}$. Gel electrophoresis was performed at $\sim 6 \mathrm{~V} / \mathrm{cM}$ for $6 \mathrm{~h}$ at $4^{\circ} \mathrm{C}$. For crossover and noncrossover gel analysis, $2 \mu \mathrm{g}$ of genomic DNA was treated with both Xhol and NgoMV followed by 1D gel analysis. Enzyme digestion and 1D gel loading conditions were the same as those described above for $1 \mathrm{D}$ and $2 \mathrm{D}$ gel loading. Southern hybridization was conducted using ${ }^{32} \mathrm{P}$-dCTP-labeled radioactive nucleotides reacted with a random primer labeling mixture (Agilent Technologies, USA). Radioactive signals were observed using a Bio-Rad phosphoimager and quantified using Quantity One (Bio-Rad, USA).

\section{Meiotic division curves}

Cells from SPM cultures were harvested and fixed in $40 \%$ ethanol containing $0.1 \mathrm{M}$ sorbitol. To count the cells undergoing meiosis I and II, DAPI stock solution was added to each cell fraction. Nuclei stained with DAPI (approximately 200 cells per each time point) were counted under a fluorescence microscope as previously described (Kim et al., 2010).

\section{RESULTS AND DISCUSSION}

\section{System for physical analysis of meiotic recombination}

The progression of meiotic recombination was monitored by one-dimensional (1D) or two-dimensional (2D) agarose gel electrophoresis (Fig.1; Hunter and Kleckner, 2001; Kim et al., 2010; Oh et al., 2007). Specifically, programmed DSB is initiated at the HIS4LEU2 locus only once in this position, and contains restriction polymorphisms to distinguish between the "Maternal" and "Parental" alleles as well as between meiotic recombination products (Fig. 1A). To obtain genomic DNA, the cells cultured in sporulation media were harvested at specific time points. The phenol-guanidine preparation method (Hong et al., 2013b; 2015; Kim et al., 2010; Koszul et al., 2008; Lee et al., 2015a) was used to prepare genomic DNA by Psoralen and UV cross-linking procedures, which involved treating each cell sample with psoralen and UV light exposure to fix the DNA interstrands. DNA was digested with the Xhol restriction enzyme and then subjected to $1 \mathrm{D}$ gel and 2D gel electrophoresis 


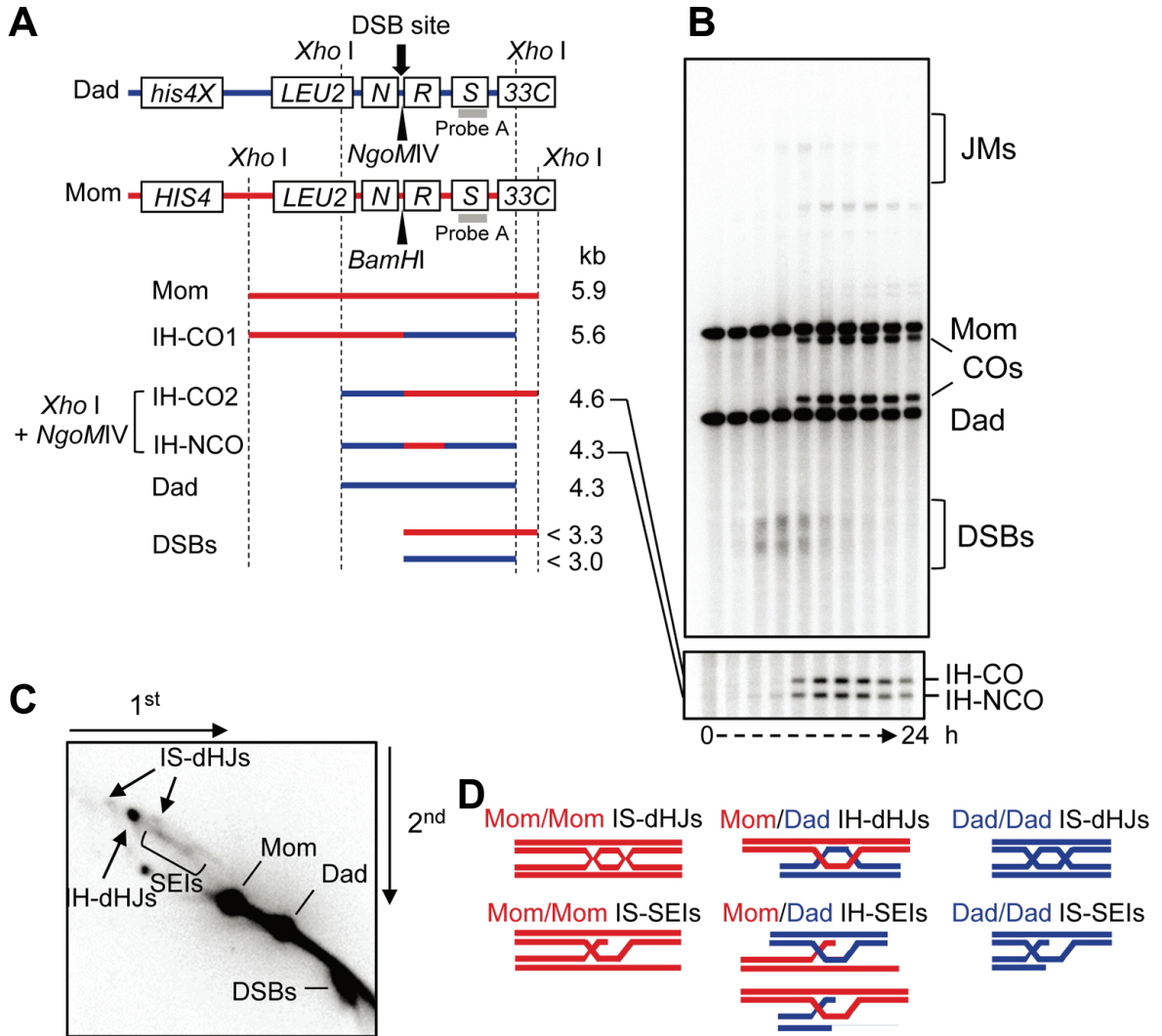

Fig. 1. Physical assay system for meiotic recombination. (A) Map of HIS4LEU2 locus (Kim et al., 2010) with $X$ hol restriction enzyme sites and probe A position is shown below. "Dad" and "Mom" chromosomes were distinguished by $\mathrm{XhOl}$ restriction polymorphism. DSB, double-strand break; $\mathrm{CO}$, crossover; $\mathrm{IH}-\mathrm{CO}$, interhomolog crossover; IH-NCO, interhomolog noncrossover. (B) 1D gel image of southern blot analysis in wild-type cells. Parental (Mom and Dad), COs, and DSBs shown in gel are described as in the figure. IH-COs and $\mathrm{IH}-\mathrm{NCO}$ were assayed using a HIS4LEU2 locus containing BamHI (Mom) and NgoMN (Dad) sites. (C) Images of native/native two-dimensional gel analysis showing joint molecules. (D) Structures of SEls and dHJs in meiotic recombination. IS-SEls, inter-sister single-end invasion; IH-SEls, interhomolog single-end invasion. IS-dHJs, inter-sister-double Holliday junctions; $\mathrm{IH}-\mathrm{dHJ}$, interhomolog double-Holliday junctions. followed by Southern hybridization analysis to detect recombination intermediates and products (Figs. 1B and 1C). Meiotic recombination eventually produces $\mathrm{IH}-\mathrm{COs}$ or interhomolog noncrossover (IH-NCO) without exchanging flanking genes (Fig. 1B; Börner et al., 2004; Hong et al., 2013; Kim et al., 2010). After digestion with both $\mathrm{Xhol}$ and $\mathrm{NgOMV}, \mathrm{IH}-\mathrm{COs}$ and $\mathrm{IH}$ NCOs from the HIS4LEU2 locus were found to be $4.6 \mathrm{~kb}$ and $4.3 \mathrm{~kb}$, respectively (Figs. $1 \mathrm{~A}$ and $1 \mathrm{~B}$ ). $\mathrm{IH}$ and IS joint molecules (SEls and dHJs) at the HIS4LEU2 locus showed different molecular weights and shapes, enabling differentiation between the SEI and $\mathrm{dHJ}$ stages by $2 \mathrm{D}$ gel electrophoresis (Figs. 1C and 1D; Lee et al., 2015b; Hong et al., 2013a; Hunter and Kleckner, 2001; Kim et al., 2010).

\section{Meiotic DSB repair is defective in the absence of Hop2 and Sae3}

The absence of Hop2 and Sae3 eliminates homologous chromosome synapsis and causes defects in DSB repair during meiotic recombination (Petukhova et al., 2003; Neale and Keerey, 2006). In a previous in vitro study, Hop2 showed distinct activity; the Hop2-Mnd1 complex stimulated Dmc1 and Rad51 recombinase activity, and purified Hop2 promoted strand invasion (Pezza et al., 2014). The Mei5-Sae3 complex interacts with Dmc1 to promote assembly of $3^{\prime}$ ssDNA on one side of a DSB (Gerton and Hawley, 2005). The physical analysis DNA in meiotic recombinant structures at the HIS4LEU2 locus has been used to examine the functions of Hop2 and Sae3 during meiotic recombination and their relationships with Mek1 kinase activity to understand the partner choice pathway. DSB formation in hop2 $\Delta$ and sae $3 \Delta$ mutants was analyzed by
1D gel electrophoresis (Fig. 2A). We further introduced the mek1as allele that is inactivated Mek1 kinase activity in the presence of 1-NA-PP1. All hop2 4 mek1as(-IN) and sae3A mek1as(-IN) cells showed defective DSB turnover, and DSB levels accumulated up to $36 \%$ compared to the control at $10 \mathrm{~h}$, and hyper-resected DSBs were also observed (Fig. 2). These results suggest that Hop2 and Sae3 are essential for promoting the DSB-to-SEI transition during meiosis. However, in the presence of a chemical inhibitor (Mek1 kinase inactivation), DSBs were efficiently processed, but there was a large number of hyper-resected DSB. IH-CO levels were not effectively increased compared to wild-type levels. Additionally, this result implies that Mek1 kinase inactivation is associated with the progression of DSB repair, but $\mathrm{IH}-\mathrm{CO}$ levels were not detectable (Fig. 2). Based on these results, we suggest that Hop2 and Sae3 are necessary for DSB repair during meiotic recombination.

Hop2 and Sae3 are involved in meiotic progression after DSB formation

DSBs were analyzed in the dmc1 $\Delta$ mek1as, hop2 $\Delta$ mek1as, and sae3A mek1as strains at the HIS4LEU2 locus where programmed DSBs are produced at one site (Figs. 1 and 3). As essential mediators, Exo1 and Mre11 together with Dna2 execute meiotic DSB end resection, specifically in the absence of Dmc1 (Garcia et al., 2011; Hong et al., 2013a; Lukaszewicz et al., 2015; Zakharyevich et al., 2010). dmc1 $\Delta$ displays DSB accumulation and hyper-resection (Fig. 3A), whereas in dmc14 mek1as $(+\mathrm{IN})$ strains, significantly reduced DSBs were observed in 2D gel analysis. Since Dmc1 plays a role in meiotic 
A

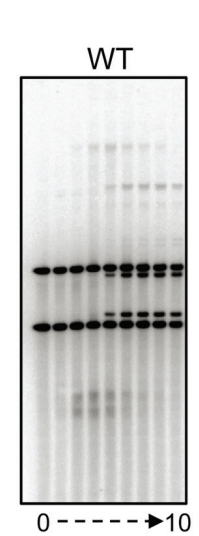

$$
\text { hop24 }
$$
mek1as $(-\mathrm{IN})$

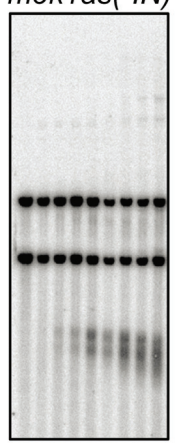

$0----\rightarrow 10$ hop24

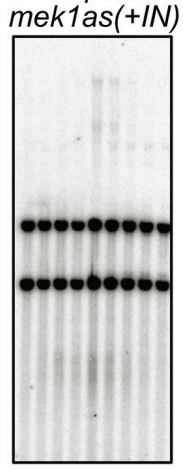

sae34
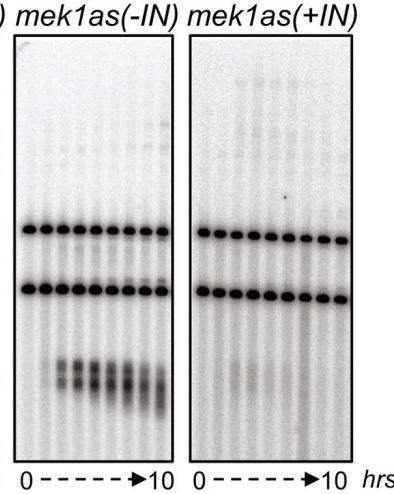

B

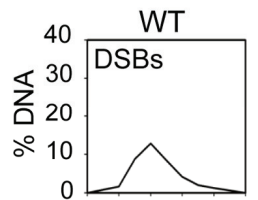

hop24

hop24

sae34

sae34
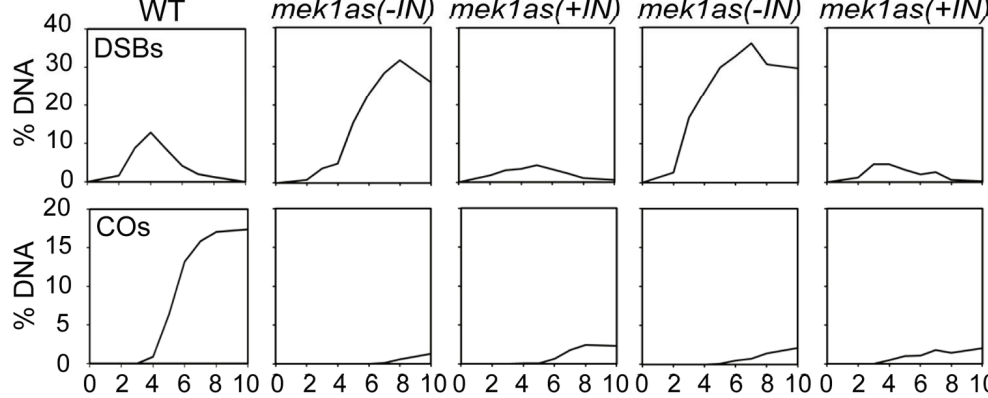

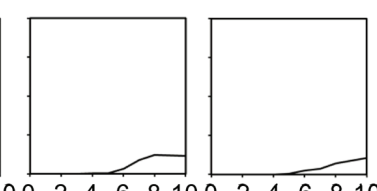

Time in SPM (h)
Fig. 2. Analysis of CO and DSB formation in hop2A mek1as and sae34 mek1as strains. (A) 1D gel analysis of DSB and CO in hop2 $\triangle$ mek1as and sae34 mek1as strains. Synchronous meiosis was induced in SPM medium in the presence or absence of 1-NA-PP1. (-IN), absence of 1-NA-PP1; $(+\mathrm{IN})$, presence of 1-NA-PP1, Mom, mom species; Dad, dad species; COs, crossover species; DSBs, double-strand breaks. (B) Quantitative analysis of DSB and $\mathrm{CO}$ in the meiotic cultures. Percentage of each DNA species versus total hybridizing DNA signals is plotted.
DSB repair, Rad51 critically functions in mitotic DSB repair in the absence of Mek1 kinase activity (Hong et al., 2013a). When Mek1 kinase was active in hop2 $\triangle$ mek1as(-IN) and sae3s mek1as(-IN), DSB resection patterns resulted in hyperresection of the DSB ends, leading to the production of extensive single-strand DNA tails at the $3^{\prime}$ end over time, as observed by 2D gel electrophoresis (Fig. 3). In the presence of Mek1 kinase activity, DSB levels remained elevated above the background signals detected in the wild-type strain because of a deficiency in DSB repair, causing DSB accumulation to higher stead-state levels. In contrast to mek1as $(+\mathrm{IN})$, where Mek1 kinase was inactive, hop2 $\Delta$ mek1as(-IN) and sae3s mek1as(-IN) showed early DSB hyper-resection, reaching maximum levels approximately 8-10 $\mathrm{h}$ into meiosis; DSBs were processed after 8 h (Fig 3B). Thus, Mek1 kinase inhibits DSB repair in the absence of Hop2 and Sae3, but when Mek1 kinase is inactivated, the cells progress efficiently into a DSB repair state via a pathway other than $\mathrm{IH}$-CO progression (Figs. 2 and 4).

\section{Hop2 and Sae3 are not required for joint molecule formation in the absence of Mek1 kinase activity} Hop2 plays a role in the DSB-to-SEl transition through Dmc1mediated strand invasion. Hop2 is a meiosis-specific protein that localizes to the chromosomes to prevent joining between nonhomologous partners and further promote joining with a homologous partner (Pezza et al., 2007). Moreover, Hop2 functions as a heterodimer complex with Mnd1 to recognize partner template DNA and to mediate its repair during meiosis, particularly by homologous paring (Chan et al., 2014; Leu et al., 1998; Tsubouchi and Roeder, 2002). Sae3 is also an essential accessory factor that forms hetero-complex with Mei5 to assist
Dmc1 to promote the meiotic DSB repair process (Hayase et al., 2004; Tsubouchi and Roeder, 2004). We investigated SEI and $\mathrm{dHJ}$ formation in wild-type, dmc1 $\Delta$ mek1as, hop2 $\Delta$ mek1as, and sae3 $\Delta$ mek1as strains that were inactivated in the presence of I-NA-PPI inhibitor (Fig. 4). The wild-type cells showed $1.4 \%$ of $\mathrm{IH}-\mathrm{dHJ}$ and $0.25 \%$ of IS-dHJ, and the ratio of $\mathrm{IH}: \mathrm{IS}-\mathrm{dHJ}$ was approximately $5: 1$. In the $d m c 1 \Delta$ mek1as(-IN) strain, both $\mathrm{IH}-\mathrm{dHJ}$ and IS-dHJ were not detected in cells, whereas in dmc14 mek1as $(+\mathrm{IN})$, the $\mathrm{IH}-\mathrm{dHJ}$ levels peaked at $0.3 \%$ but IS$\mathrm{dHJ}$ remained undetected (Fig. 4A and 4B). In the hop2 $\triangle$ mek1as(-IN) strain, $\mathrm{IH}-\mathrm{dHJ}$ levels were approximately $0.1 \%$, but IS-dHJs were undetectable in cells; whereas hop2 $\Delta$ mek1as $(+\mathrm{IN})$ showed $\sim 0.1 \% \mathrm{IH}-\mathrm{dHJ}$ and $\sim 1 \% \mathrm{IS}-\mathrm{dHJ}$, with a ratio of $\mathrm{IH}-\mathrm{dHJ}$ to IS-dHJ approximately 1:9 (Fig. 4C and 4D; Supplementary Table 2). In the sae3 $\triangle$ mek1as(-IN) strain, cells exhibited $0.1 \% \quad \mathrm{IH}-\mathrm{dHJ}$ and $0.25 \% \quad \mathrm{IS}-\mathrm{dHJ}$, whereas sae34 mek1as(+IN) cells displayed close to $0 \% \mathrm{IH}-\mathrm{dHJ}$ and $0.75 \% \mathrm{IS}-$ $\mathrm{dHJ}$ levels (Figs. 4C and 4D). Thus, Mek1 kinase inactivation triggered DSB repair and JM formation by converting the recombinational mode from $\mathrm{IH}$ bias to IS bias. Further, in mek1as $(+\mathrm{IN})$ cells, SEls appeared to form inter-sister arcs of SEI going to IS-dHJ (Fig. 4; Kim et al., 2010). Taken together, these results indicate that when Mek1 kinase was inactivated, cells underwent DSB repair through the inter-sister crossover (IS-CO) pathway. Thus, Mek1 kinase regulates recombination in the functional stages of Hop2 and Sae3 to convert from the mitotic mode into the meiotic mode.

Both Hop2 and Sae3 are required for the production of interhomolog recombination products

Homologous recombination leads to $\mathrm{CO}$ or $\mathrm{NCO}$, the outcomes 
A

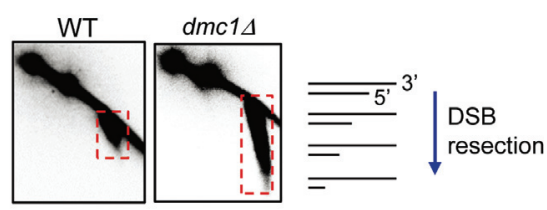

B

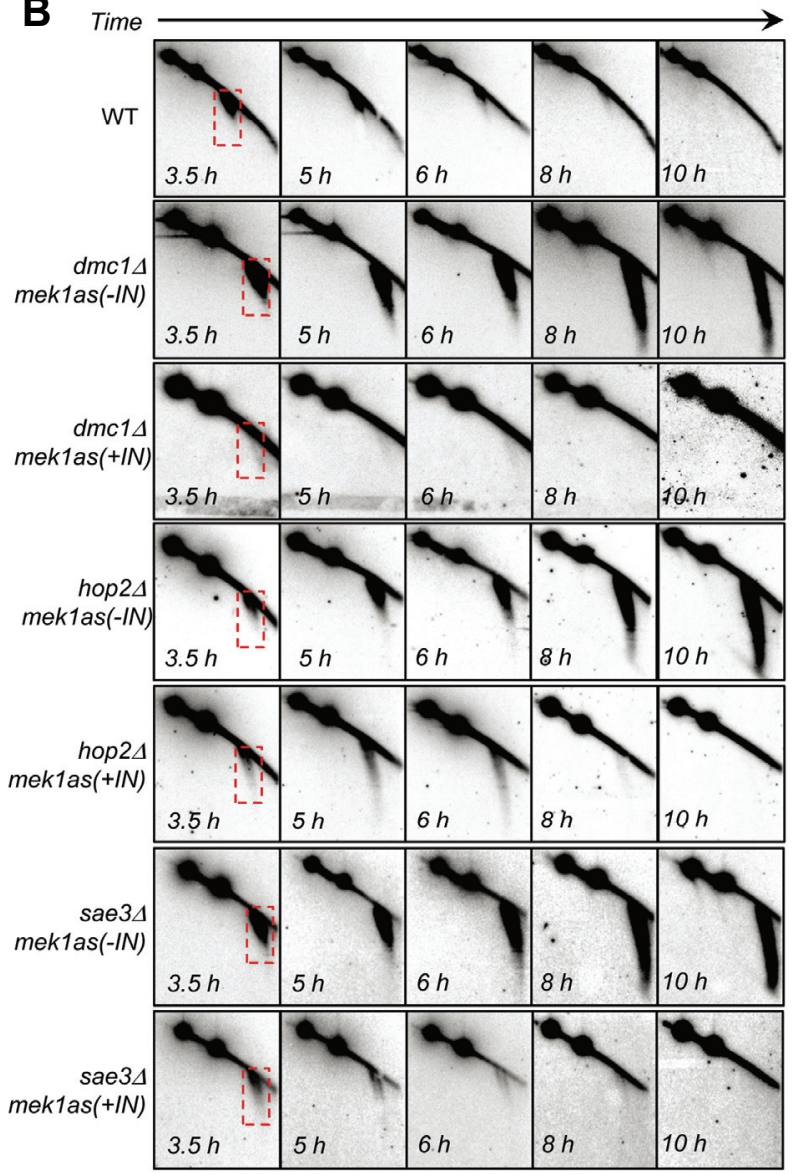

Fig. 3. DSB resection in WT, dmc1 14 mek1as, hop2 $\Delta$ mek1as, and sae34 mek1as strains. (A) Representative images marking DSB resection location by two-dimensional gel analysis. (B) DSB resection patterns at each time point. Dot boxes indicate DSB area of the 2D gel.

of meiotic DSB repair, in the flanking region of paired chromosomes (Allers et al., 2001; Mancera et al., 2008; Martini et al., 2006;). CO and NCO products can be distinguished at the HIS4LEU2 locus after digestion with Xhol and NgoMV. In wildtype cells, the maximum levels of $\mathrm{CO}$ and $\mathrm{NCO}$ were $3.8 \%$ and $3.2 \%$, respectively. However, in the hop2 $\Delta$ mek1as and sae3 mek1as strains, regardless of whether 1-NA-PP1 inhibitor was present, the $\mathrm{CO}$ and $\mathrm{NCO}$ levels were less than 0.3\% (Figs. 5A and 5B). Because hop2 $\triangle$ mek1as $(+\mathrm{IN})$ and sae3 $\Delta$ mek1as $(+\mathrm{IN})$ exhibit DSB hyper-resection and accumulation (Fig. 3B) without ongoing repair, low levels of $\mathrm{COs}$ and $\mathrm{NCO}$ are expected. In contrast, DSBs in the hop2 4 mek1as $(+\mathrm{IN})$ and sae34 mek1as $(+\mathrm{IN})$ strains were repaired as in the wild-type strain (Fig. 3B) and the IS-dHJ level was highly increased compared to levels in cells without a 1-NA-PP1 inhibitor. Thus, since 1D gel analysis can only detect $\mathrm{IH}-\mathrm{COs}$ or $\mathrm{IH}-\mathrm{NCOs}$,
A
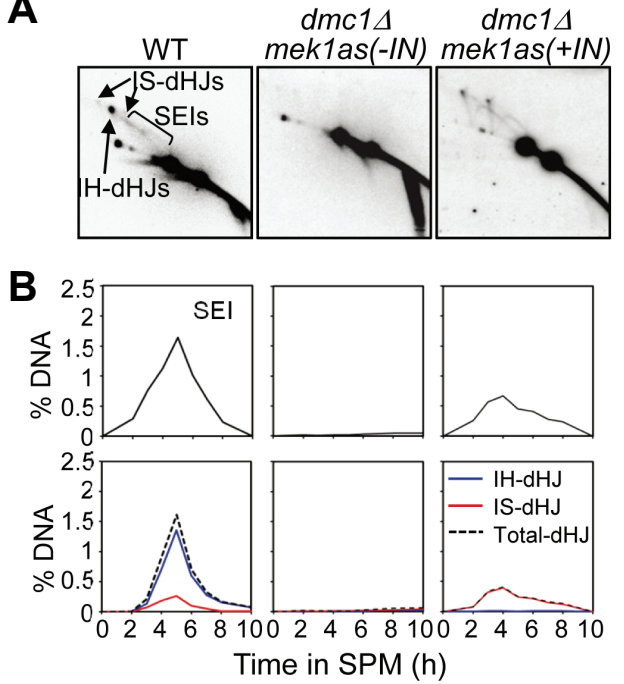

C

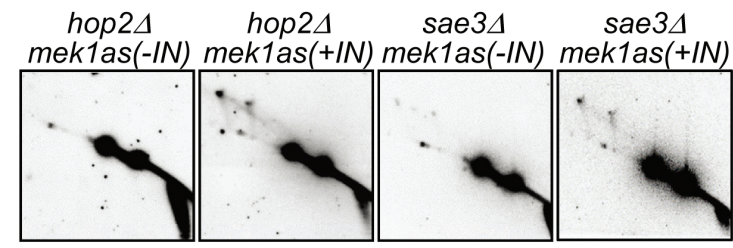

D

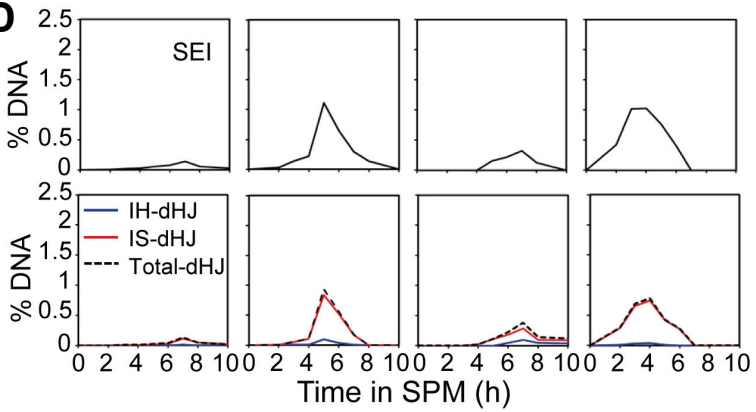

Fig. 4. Analysis of $\mathrm{dHJ}$ and SEl formation in WT, dmc1 $1 \Delta$ mek1as, hop2 $\Delta$ mek1as, and sae34 mek1as strains in the presence of absence of 1-NA-PP1. (A) Representative images of 2D gel analysis in WT and dmc1 $1 \Delta$ mek1as strains. (B) Quantitative analysis of SEls and $\mathrm{dHJs}$ from two-dimensional gel images. Dot lines represent total $\mathrm{dHJ}$ levels. (C) Representative images of 2D gel analysis in hop2 $\Delta$ mek1as and sae34 mek1as strains. (D) Quantitative analysis of SEls and dHJs from two-dimensional gel images. Dot lines represent total $\mathrm{dHJ}$ levels.

DSBs processed into IS-dHJ were resolved to IS-COs or ISNCOs (Fig. 5C). This explains why DSB repair enters the mitotic mode, which uses sister templates rather than homologous templates, as in meiotic mode. Hence, Hop2 and Sae3 play roles in the fate of meiotic DSBs and determine whether programmed DSBs are processed by homolog bias or sister bias by Mek1 kinase. When Mek1 kinase activity is inhibited, the meiotic DSB repair procedure is directed towards the sister bias mechanism that use sister chromatids as templates (Tracy et al., 2010). In this case, DSBs are processed in the mitotic mode and are finally resolved with IS-NCO or IS-CO. However, more precise methods are required to distinguish these products. If 
A

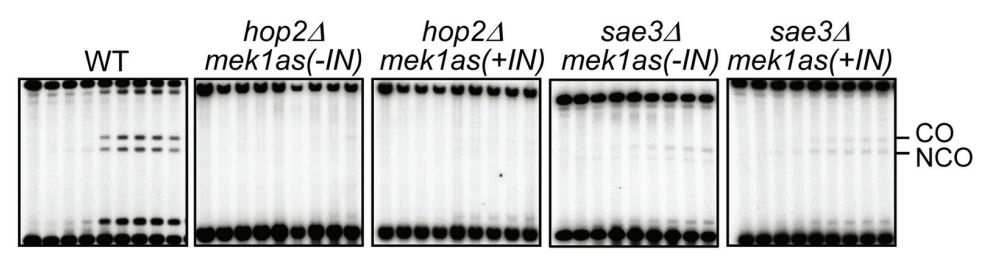

B

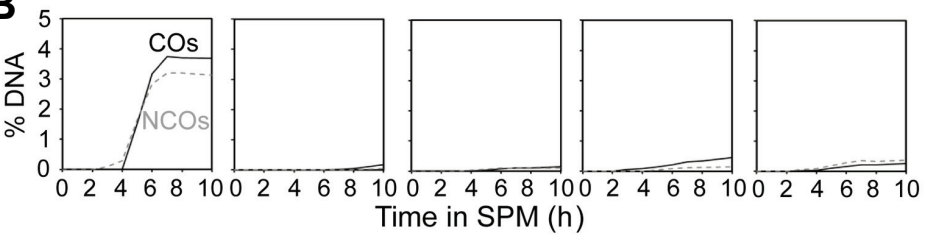

Fig. 5. $\mathrm{CO}$ and $\mathrm{NCO}$ formation in WT, hop2 $\triangle$ mek1as, and sae34 mek1as strains. (A) Representative one-dimensional physical analysis gel images of WT, hop2 $\Delta$ mek1as, and sae34 mek1as strains. (B) Quantitative analysis graphs showing $\mathrm{COs}$ and NCOs from one-dimensional gel images. (C) Proposed model for the roles of Hop2 and Sae3 in interhomolog recombination during meiosis.

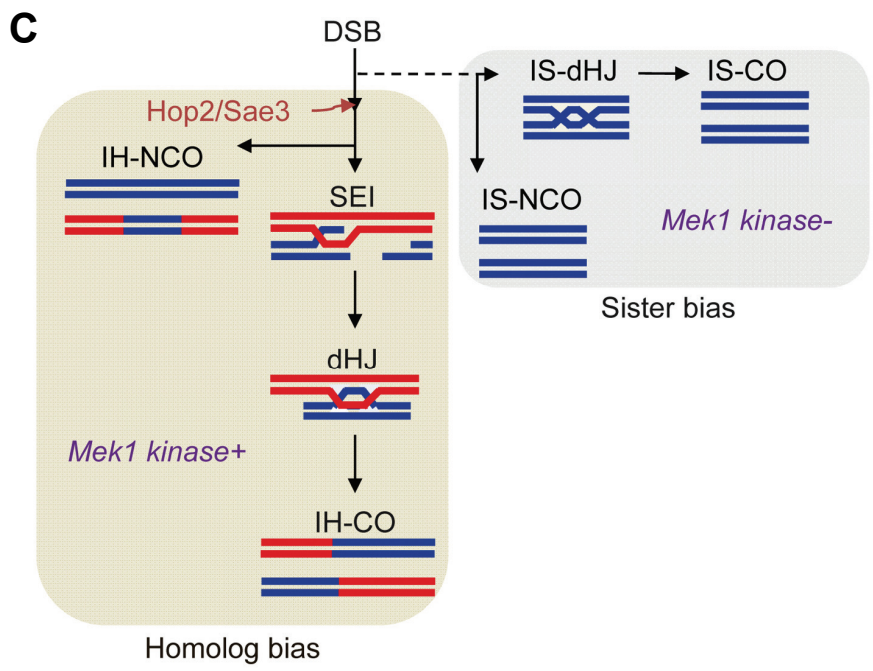

Mek1 kinase plays the same role as in wild-type cells, the default option for programmed DSB repair is homologous bias, which uses homologous chromatids for recombination. When cells undergo defects in the SEI-to-dHJ transition, some of the SEls may be converted into $\mathrm{IH}-\mathrm{NCO}$ products, while SEI is stably converted into an $\mathrm{IH}-\mathrm{CO}$ product when maintenance is sustained. Both Hop2 and Sae3 function during early prophase I before the SEI step as accessory factors of Dmc1, and therefore programmed DSBs are hyper-resected and accumulate because when subsequent pathways are blocked, such as in the absence of Hop2 or Sae3 (Fig. 5C).

In most organisms, recombinational interactions precisely occur between homologs to promote the efficient pairing of chromosomes and synapsis formation during meiosis. Dmc1 is a key factor in meiotic recombination and is required for homolog bias in the intrinsic pathway in meiosis. The budding yeast proteins Hop2 and Sae3 are assembled on DNA to promote heteroduplex DNA formation in a manner that affects Dmc1 strand exchange activity during meiotic recombination. Dmc1 and its accessory factors, Hop2 and Sae3, ensure that the first DSB ends are properly assembled onto the partner template to form a D-loop and that stably form SEI, which gives rise to the $\mathrm{dHJ}$. These results suggest that during the DSB repair pathway, an early decision between the interhomolog and inter-sister pathway occurs, with most inter-sister $\mathrm{CO}$ arising from sister bias, and interhomolog $\mathrm{CO}$ events progressing to form an $\mathrm{IH}$ $\mathrm{dHJ}$. Further, IH pathway is switched to IS pathway when Mek1 kinase becomes inactive (Hong et al., 2013b). Thus, these results indicate that Hop2 and Sae3 require Mek1 kinase activity, which also plays a role in the post-DSB stage to promote Dmc1-mediated meiotic recombination.

Note: Supplementary information is available on the Molecules and Cells website (www.molcells.org).

\section{ACKNOWLDEGMENTS}

This research was supported by grants from the National Research Foundation of Korea funded by the Ministry of Science, ICT \& Future Planning (2012M3A9C6050367; 2014R1A2A1A 11051584; 2015R1A2A1A15054378).

\section{REFERENCES}

Allers, T., Lichten, M. (2001). Differential timing and control of noncrossover and crossover recombination during meiosis. Cell 106, 47-57.

Börner, G.V., Kleckner, N., and Hunter, N. (2004). Crossover/noncrossover differentiation, synaptonemal complex formation, and regulatory surveillance at the leptotene/zygotene transition of meiosis. Cell 117, 29-45.

Busygina, V., Sehorn, M.G., Shi, I.Y., Tsubouchi, H., Roeder, G.S., 
and Sung, P. (2008). Hed1 regulates Rad51-mediated recombination via a novel mechanism. Genes Dev. 22, 786-795.

Cannavo, E., and Cejka, P. (2014). Sae2 promotes dsDNA endonuclease activity within Mre11-Rad50-Xrs2 to resect DNA breaks. Nature 514, 122-125.

Chan, Y.L., Brown, M.S., Qin, D., Handa, N., and Bishop, D.K. (2014). The third exon of the budding yeast meiotic recombination gene HOP2 is required for calcium-dependent and recombinase Dmc1-specific stimulation of homologous strand assimilation. J. Biol. Chem. 289, 18076-18086.

Chi, P., San Filippo, J., Sehorn, M.G., Petukhova, G.V., and Sung, P. (2007). Bipartite stimulatory action of the Hop2-Mnd1 complex on the Rad51 recombinase. Genes Dev. 21, 1747-1757.

Ferrari, S.R., Grubb, J., and Bishop, D.K. (2009). The Mei5-Sae3 protein complex mediates Dmc1 activity in Saccharomyces cerevisiae. J. Biol. Chem. 284, 11766-11770.

Garcia, V., Phelps, S.E., Gray, S., and Neale, M.J. (2011). Bidirectional resection of DNA double-strand breaks by Mre11 and Exo1. Nature 479, 241-244.

Gerton, J.L., and Hawley, R.S. (2005). Homologous chromosome interactions in meiosis: diversity amidst conservation. Nat. Rev. Genet. 6, 477-487.

Hayase, A., Takagi, M., Miyazaki, T., Oshiumi, H., Shinohara, M., and Shinohara, A. (2004). A protein complex containing Mei5 and Sae3 promotes the assembly of the meiosis-specific RecA homolog Dmc1. Cell 119, 927-940.

Henry, J.M., Camahort, R., Rice, D.A., Florens, L., Swanson, S.K., Washburn, M.P., and Gerton, J.L. (2006). Mnd1/Hop2 facilitates Dmc1-dependent interhomolog crossover formation in meiosis of budding yeast. Mol. Cell. Biol. 26, 2913-2923.

Hong, S., Sung, Y., Yu, M., Lee, M., Kleckner, N., and Kim, K.P. (2013a). The logic and mechanism of homologous recombination partner choice. Mol. Cell 51, 440-453.

Hong, S., and Kim, K.P. (2013b). Shu1 Promotes Homolog Bias of Meiotic Recombination in Saccharomyces cerevisiae. Mol. Cells $36,446-454$

Hong, S., Choi, E.H., and Kim, K.P. (2015). Ycs4 is required for efficient double-strand break formation and homologous recombination during meiosis. J. Microbiol. Biotechnol. 25, 1026-1035.

Hunter, N., and Kleckner, N. (2001). The single-end invasion: an asymmetric intermediate at the double-strand break to doubleholliday junction transition of meiotic recombination. Cell 106, 5970.

Kang, H.A., Shin, H.C., Kalantzi, A.S., Toseland, C.P., Kim, H.M., Gruber, S., Peraro, M.D., and Oh, B.H. (2015). Crystal structure of Hop2-Mnd1 and mechanistic insights into its role in meiotic recombination. Nucleic Acids Res. 43, 3841-3856.

Keeney, S. (2001). Mechanism and control of meiotic recombination initiation. Curr. Topics Dev. Biol. 52, 1-53.

Kim, K.P., Weiner, B.M., Zhang, L., Jordan, A., Dekker, J., and Kleckner, N. (2010). Sister cohesion and structural axis components mediate homolog bias of meiotic recombination. Cell 143, 924-937.

Koszul, R., Kim, K.P., Prentiss, M., Kleckner, N., and Kameoka, S. (2008). Meiotic chromosomes move by linkage to dynamic actin cables with transduction of force through the nuclear envelope. Cell 133, 1188-1201.

Lao, J.P., Cloud, V., Huang, C.C., Grubb, J., Thacker, D., Lee, C.Y., Dresser, M.E., Hunter, N., and Bishop, D.K. (2013). Meiotic crossover control by concerted action of Rad51-Dmc1 in homolog template bias and robust homeostatic regulation. PLoS genetics 9, e1003978.

Lee, M.S., Yu, M., Kim, K.Y., Park, G.H., Kwack, K.B., and Kim, K.P. (2015a). Functional validation of rare human genetic variants involved in homologous recombination using Saccharomyces cerevisiae. PLoS One 10, e0124152.
Lee, M.S., Yoon, S.W., and Kim, K.P. (2015b). Mitotic cohesin subunit Mcd1 regulates the progression of meiotic recombination in budding yeast. J. Microbiol. Biotechnol. 25, 598-605.

Leu, J.Y., Chua, P.R., and Roeder, G.S. (1998). The meiosisHop2 protein of S. cerevisiae ensures synapsis between homologous chromosomes. Cell 94, 375-386.

Lukaszewicz, A., Shodhan, A., and Loidl, J. (2015). Exo1 and Mre11 execute meiotic DSB end resection in the protist Tetrahymena. DNA Repair 35, 137-143.

Mancera, E., Bourgon, R., Brozzi, A., Huber, W., Steinmetz, L. M. (2008). High-resolution mapping of meiotic crossovers and noncrossovers in yeast. Nature 454, 479-485.

Martini, E., Diaz, R. L., Hunter, N., Keeney, S. (2006). Crossover homeostasis in yeast meiosis. Cell 156, 285-95.

Neale, M.J., and Keeney, S. (2006). Clarifying the mechanics of DNA strand exchange in meiotic recombination. Nature 442 153-158.

Niu, H., Li, X., Job, E., Park, C., Moazed, D., Gygi, S.P., and Hollingsworth, N.M. (2007). Mek1 kinase is regulated to suppress double-strand break repair between sister chromatids during budding yeast meiosis. Mol. Cell. Biol. 27, 5456-5467.

Niu, H., Wan, L., Busygina, V., Kwon, Y., Allen, J.A., Li, X., Kunz, R.C., Kubota, K., Wang, B., Sung, P., et al. (2009). Regulation of meiotic recombination via Mek1-mediated Rad54 phosphorylation. Mol. Cell 36, 393-404.

Oh, S.D., Lao, J.P., Hwang, P.Y., Taylor, A.F., Smith, G.R., and Hunter, N. (2007). BLM ortholog, Sgs1, prevents aberrant crossing-over by suppressing formation of multichromatid joint molecules. Cell 130, 259-272.

Petukhova, G.V., Romanienko, P.J., Camerini-Otero, R. D. (2003). The Hop2 protein has a direct role in promoting interhomolog interactions during mouse meiosis. Dev. Cell 5, 927-936.

Petukhova, G.V., Pezza, R.J., Vanevski, F., Ploquin, M., Masson, J.Y., and Camerini-Otero, R.D. (2005). The Hop2 and Mnd1 proteins act in concert with Rad51 and Dmc1 in meiotic recombination. Nat. Struct. Mol. Biol. 12, 449-453.

Pezza, R.J., Voloshin, O.N., Vanevski, F., and Camerini-Otero, R.D. (2007). Hop2/Mnd1 acts on two critical steps in Dmc1-promoted homologous pairing. Genes Dev. 21, 1758-1766.

Pezza, R.J., Voloshin, O.N., Volodin, A.A., Boateng, K.A., Bellani, M.A., Mazin, A.V., and Camerini-Otero, R.D. (2014). The dual role of HOP2 in mammalian meiotic homologous recombination. Nucleic Acids Res. 42, 2346-2357.

Schwacha, A., and Kleckner, N. (1997). Interhomolog bias during meiotic recombination: meiotic functions promote a highly differentiated interhomolog-only pathway. Cell 90, 1123-1135.

Shinohara, A., Gasior, S., Ogawa, T., Kleckner, N., and Bishop, D. (1997). Saccharomyces cerevisiae recA homologues RAD51 and $\mathrm{DMC} 1$ have both distinct and overlapping roles in meiotic recombination. Genes Cells 10, 615-629.

Tracy, L.C., and Nancy, M.H. (2010). Mek1 suppression of meiotic double-strand break repair is specific to sister chromatids, chromosome autonomous and independent of Rec8 cohesin complexes. Genetics 185, 771-782.

Tsubouchi, H., and Roeder, G.S. (2002). The Mnd1 protein forms a complex with hop2 to promote homologous chromosome pairing and meiotic double-strand break repair. Mol. Cell. Biol. 22, 30783088.

Tsubouchi, H., and Roeder, G.S. (2004). The budding yeast mei5 and sae3 proteins act together with dmc1 during meiotic recombination. Genetics 168, 1219-1230.

Zakharyevich, K., Ma, Y., Tang, S., Hwang, P.Y., Boiteux, S., and Hunter, N. (2010). Temporally and biochemically distinct activities of Exo1 during meiosis: double-strand break resection and resolution of double Holliday junctions. Mol. Cell 40, 1001-1015. 